\title{
WANDA REWIEŃSKA (1897-1942): PRÉCURSEUR DE LA THÉORIE DE CHRISTALLER DANS LA GÉOGRAPHIE ÉCONOMIQUE POLONAISE
}

Wanda Rewieńska, de l'Université Stefan Batory de Wilno, mérite certainement d'être considérée comme un des plus éminents représentants de la géographie économique en Pologne. Elle appartenait à la nouvelle génération de géographes, formés déjà en Pologne indépendante et engagés dans les études liées à la nouvelle réalité socio-économique.

La géographie de l'habitat était au centre de ses préoccupations; elle s'intéresait aussi à l'anthropogéographie et à la géographie des transports. Les problèmes propres à ces différentes matières se chevauchent dans ses études, et se complètent, créant un ensemble d'oeuvres étonnant par l'ampleur du regard et par la précision de méthodes utilisées.

La manière d'aborder l'étude des "Isochrones de Wilno", premier travail de Wanda Rewieńska publié en 1929, est aussi caractéristique de ses recherches ultérieures. En effet, elle considère le transport comme un des principaux facteurs d'intégration du réseau d'habitat de la région. Le transport contribue à l'intégration des localités, car, d'après elle, "ce n'est pas seulement la distance réelle, mais la distance-temps qui décide de l'échange des valeurs culturelles et d'une meilleure unification de l'État".

Jusque-là, les villes du Nord-Est de la Pologne se développaient vers les périphéries; ce n'est qu'après l'indépendance en 1918 que, selon Wanda Rewieńska, se créent des sortes de "foyers secondaires de développement". Cette approche peut sans doute être considérée comme l'un de premiers essais d'identification des pôles de développement - notion utilisée dans la description et dans l'explication du développement socio-économique. Cette conception, on le sait, a été pleinement exposée dans les travaux de F. Perrous.

L'approche anthropogéographique dans l'étude des problèmes sociaux et économiques caractérise les travaux sur la géographie de l'habitat, entrepris par Wanda Rewieńska au début des années trente. Elle se réfere à l'optique de la géographie humaine française de P. Vidal de la Blache, qui, en acceptant les lignes principales de l'anthropogéographie de F. Ratzel, les a profondément modifiées. L'idée de l'unité matérielle de toutes les formes de la vie, de la communauté d'espace et de l'interprétation organique des 
groupes humains constitue le point de départ de Wanda Rewieńska. Accordant au milieu naturel un rôle de facteur de formation de la structure économique, contribuant aussi aux fondements culturels des communautés humaines, elle remarque néanmoins l'importance de plus en plus visible de l'activité de l'homme dans la transformation de son environnement.

Ce point de vue est pleinement exposé dans l'étude sur Bereza Kartuska (1934), qui ne se limite pas seulement à l'analyse anthropogéographique de la localité. Ses fonctions y sont étudiées, ainsi que la structure de son espace socio-économique et sa zone d'influence. Pour déterminer l'espace vital réel, Wanda Rewieńska utilise, suivant F. Uhorczak (1932), l'équidistance do $50 \mathrm{~m}$ par rapport aux maisons d'habitation de la périphérie.

L'étude de Wanda Rewieńska constitue également une des premières, sinon la première, analyse écologique de l'espace urbain, dans lequel se forment "des regroupements aux caractéristiques économiques distinctes". D'après l'analyse de la structure d'emploi, de la structure ethnique et des éléments physionomiques, Bereza Kartuska comporte ainsi six quartiers socio-économiques: commercial, industriel, administratif (d"intelligentsia"), militaire, artisanal et rural. Wanda Rewieńska aperçoit également un phénomène intéressant: les prémices du "creusement du centre", c'est-à-dire du reflux de la population du centre, si caractéristique des grandes villes. Cette remarque témoigne de l'imagination et de lintuition sociologique et urbanistique de Wanda Rewieńska: dans une petite ville, peuplée de 4521 habitants seulement en 1931, elle a perçu des processus qui ont atteint leur apogée bien après la Deuxième guerre mondiale - et ont été décrits par les géographes anglo-saxons dans les années soixante et soixante-dix.

L'étude des fonctions économiques de la ville par rapport à son arrière-pays peut aussi être considérée comme novatrice. Examinant la genèse de Bereza Kartuska, Wanda Rewieńska prouve que le principal facteur de création de la ville a été la desserte d'un vaste espace, rural et urbain. Elle étudie le développement de Bereza Kartuska non seulement dans le sens "horizontal" (spatial), mais aussi dans le sens "vertical" (hiérarchique) et considère que la promotion de la ville au $\mathrm{XX}^{\mathrm{e}}$ siècle était étroitement liée à sa promotion dans le réseau hiérarchique d'habitat.

Dans ses reflexions sur la hiérarchie des villes, Wanda Rewieńska est proche des conceptions théoriques de W. Christaller (1933), ou même de celles d'A. Lösch (1940). En effet, dans son interprétation, une ville est un centre principal de la vie économique et sociale pour "la population rassemblée dans un point du globe", et les bourgs comme celui de Bereza Kartuska le sont "pour les localités rurales dispersées autour". Le degré dans la hiérarchie des localités est proportionnel au rayon de leur influence. Dans le cas de Bereza Kartuska ce rayon ne dépasse pas $50 \mathrm{~km}$ env. Cela correspondrait donc à la localité de sixième rang (de type $\mathrm{P}$ dans le modèle de $\mathrm{W}$. Christaller), desservant un espace de $11000 \mathrm{~km}^{2}$.

Bien entendu, l'importance régionale de Bereza Kartuska dépassait 
largement ses possibilités réelles, car dans la conception de W. Christaller une telle fonction ne peut être assurée que par une localité de 100000 habitants. Rappelons donc qu'il s'agissait d'une région de frontière, au réseau d'habitat dispersé et incohérent, dépourvu non seulement de centres régionaux bien structurés, mais même de centres locaux.

Une place centrale dans l'oeuvre de Wanda Rewieńska revient à l'étude intitulée "Villes et bourgs dans le Nord-Est de la Pologne" (1938), sorte de synthèse sur le réseau d'habitat dans cette région de la Pologne. L'accent est mis sur la hiérarchie des villes, sur le rôle qu'elles exercent dans le réseau d'habitat. "Il existe des règles qui commandent la structure du réseau des villes et la hiérarchie développée en son sein". Les petites localités ont moins d'importance et occupent une position inférieure dans l'ensemble; cette importance et cette position grandissent avec la grandeur de la ville.

Selon Wanda Rewieńska, et selon W. Christaller (1933), l'ordre élémen-taire de la réalité sociale réside dans la disposition de centres, dans laquelle le centre exerce le même rôle que celui du noyau par rapport à la matière qui l'entoure. Pour Christaller, les signes extérieurs de cet ordre central sont des établissements comme une église, un hôtel de ville ou une école; les villes centrales sont celles qui exercent des fonctions centrales, c'est-à-dire dans tous les domaines de l'activité qui desservent un espace plus vaste. Grâce aux fonctions centrales, une localité constitue un centre régional et un noeud de transport facilitant les contacts avec le monde extérieur.

Wanda Rewieńska remarque que dans le Nord-Est de la Pologne, les localités rurales développées de façon autonome se formaient autour des églises, des couvents, des châteaux-forts ou des manoirs. Elle mentionne ces quatre centres de cristallisation, dont certains pouvaient agir simultanément. Une localité quittait le réseau rural lorsque l'échange commençait, donc lorsque les fonctions de commerce et de service s'ajoutaient à la fonction agricole.

Un des derniers travaux de Wanda Rewieńska, "La répartition des villes et des bourgs dans le Nord-Est de la Pologne" (1939), est consacré à la recherche des relations entre l'importance, le nombre et la répartition des localités. Dans le Nord-Est de la Pologne cette répartition, au premier coup d'oeil chaotique et désordonnée, s'avère en effet logique et régie d'après "un certain schéma géométrique". Ce schéma n'a pas de caractère "linéaire" (car les localités de grandeur similaire sont réparties uniformément), mais, comme l'écrit Wanda Rewieńska, celui “de foyers": les localités de diverse importance se rassemblent autour d'un plus grand centre.

Les "centres régionaux" exercent un rôle social et économique de première importance; ils "relient tel ou tel territoire en un ensemble cohérent". Ce rôle est habituellement joué par les villes situées dans la partie centrale d'un espace de marché. Elles se créaient à l'intersection d'importantes voies de communication, où arrivaient les voyageurs et où se développait le commerce. Comme l'accès aux villages environnants y est 
facile, elles desservent parfois un vaste arrière-pays. Outre les "centres régionaux" il existe un groupe de petites localités, moins importantes économiquement, à l'accès habituellement limité. Elles desservent quelques villages environnants. Ce type de localité correspond presque exactement au type le plus inférieur dans la hiérarchie de W. Christaller (type $M$, desservant un espace de 4-7 $\mathrm{km}^{2}$ ).

Les "centres régionaux" du Nord-Est de la Pologne étaient issus des villes et bourgs, se transformant et s'adaptant aux nouvelles fonctions économiques et sociales. Notons que Wanda Rewieńska ne se limite pas à une réflexion théorique, mais identifie les localités de ce type.

L'oeuvre de Wanda Rewieńska constitue sans doute une des plus importantes réalisations de la géographie humaine polonaise de l'entre-deux-guerres. Dans ses études sur le réseau d'habitat elle tendait logiquement vers une synthèse, sous forme de modèle déductif; elle était proche des solutions que l'on peut trouver dans la théorie des lieux centraux de W. Christaller. Elle le dépassait souvent, décrivant p. ex. les transformations de la centralité par les frontières politiques, l'héritage historique, les conditions naturelles. Les résultats de ses recherches avaient également une importance pratique. Ainsi, ses études étaient utilisées dans les tentatives de rationalisation du réseau d'habitat du Nord-Est de la Pologne, région attardée dans le développement socio-économique.

Il n'a pas été donné à Wanda Rewieńska de vérifier ses hypothèses et ses modèles, construits dans les années trente à partir des riches observations rassemblées lors de nombreuses études de terrain. La guerre a interrompu ses recherches novatrices. Elle a été fusillée par les Allemands le 24 novembre 1942 lors d'une exécution de masse à Wilno.

\section{BIBLIOGRAPHE}

Ch ristall e r W., 1933, Die zentralen Orte in Süddeutschland, Gustav Fischer, Jena Lö s ch A., 1949, Die räumliche Ordnung der Wirtschaft, Gustaw Fischer, Jena

Rewieńska W., 1929, Izochrony Wilna (Les isochrones de Wilno), Tow. Przyjaciół Nauk w Wilnie, Wydział Nauk Matematycznych i Przyrodniczych, t. V, Wilno

Rewieńska W., 1934, Bereza Kartuska, wybrane rozdzialy $z$ antropogeografii miasteczka (Bereza Kartuska, quelques chapitres d'anthropogéographie d'une petite ville), Tow. Przyjaciół Nauk w Wilnie, Prace Zakładu Geologii i Geografii Uniwersytetu Stefana Batorego, Wilno

Rewieńska W., 1939, "Rozmieszczenie miast i miasteczek w północno-wschodniej Polsce" (Répartition des villes et bourgs dans le Nord-Est de la Pologne), Przeglad Geograficzny t. XVIII, Warszawa

Uhorczak F., 1932, "Z metodyki badań nad osadnictwem" (Sur les méthodes de recherches sur l'habitat), Czasopismo Geograficzne, fasc. 1-3, Lwów. 